\title{
EFEITO DO USO DE LODO DE ESGOTO NA QUALIDADE NUTRICIONAL E NA PRODUÇÃO DE FITOMASSA DA UROCHLOA DECUMBENS
}

\section{EFFECT OF THE USE OF SEWAGE SLUDGE ON NUTRITIONAL QUALITY AND PHYTOMASS OF UROCHLOA DECUMBENS}

\section{Janardelly Gomes de Souza; Daniela Berto Dourado; Marcelo Rodrigo Alves, Carlos Henrique dos Santos, Marco Aurélio Factori}

Universidade do Oeste Paulista - UNOESTE, Faculdade de Engenharia, FEP, Presidente Prudente, SP.

E-mail: marceloalves@unoeste.br

RESUMO - O uso do lodo de esgoto possibilita o reaproveitamento dos nutrientes com rendimentos equivalentes ou superiores aos conseguidos com adubos químicos, podendo contribuir para melhoria dos solos degradados. O objetivo do estudo foi avaliar a capacidade de utilização do lodo da ETE em conjunto ou mesmo como alternativa à adubação química convencional na produção da braquiaria. Dessa forma, foi montado um experimento em blocos inteiramente casualizados, com cinco tratamentos e seis repetições, onde verificou-se os parâmetros de análise química e a produção de massa foliar da braquiária. Os dados obtidos foram submetidos à análise estatística, e análises de regressão. Mediante os resultados conclui-se que a adubação orgânica se equivaleu com a adubação química.

Palavras-chave: biossólido; adubo químico; braquiaria.

\begin{abstract}
The use of sewage sludge allows the reutilization of nutrients with yields equivalent to or greater than those obtained with chemical fertilizers, and may contribute to the improvement of degraded soils. The aim of the study was to evaluate the ability of the ETE sludge to be used together or even as an alternative to conventional chemical fertilization in braquiaria production. Thus, a completely randomized block experiment was carried out, with five treatments and six replications, where the parameters of chemical analysis and leaf mass production of the braquiaria were verified. Data were submitted to statistical analysis and regression analysis. The results show that organic fertilization was equivalent to chemical fertilization.
\end{abstract}

Recebido em: $13 / 08 / 2018$ Revisado em: 03/09/2018 Aprovado em: 18/09/2018
Keywords: biosolid; chemical fertilizer; braquiaria. 


\section{INTRODUÇÃO}

Há várias alternativas para a disposição final do lodo, como por exemplo: a disposição em aterro sanitário, a recuperação de solos e áreas degradadas, uso agrícola e a disposição oceânica (TSUTIYA, 2000).

As práticas de disposição do lodo são benéficas, quando a aplicação se usufruir das propriedades do produto como fertilizante, o que fornece macro e micro nutrientes melhorando a característica em solos degradados (ANDREOLI; PEGORINI; FERNANDES, 2001), podendo contribuir ainda para a melhoria de $\mathrm{pH}$ e incita a atividade microbiana na área (ALAMINO, 2010).

A matéria orgânica influência nos aspectos físicos, químicos e biológicos do solo, sendo que a mesma depende de uma série de fatores como temperatura, aeração, $\mathrm{pH}$, disponibilidade de água e nutrientes condicionados pelo manejo dos solos (NASCIMENTO et al., 2010) podendo agir ainda como um agente cimentante promovendo a agregação de suas articulas e melhorando também a capacidade de retenção de água. De maneira geral, as adubações orgânicas aumentam a infiltração e a retenção de água no solo e a estabilidade dos agregados, tornando estes solos mais resistentes aos processos erosivos (VON SPERRLING; GONÇALVES, 2001).

A braquiária é um gênero que destacase na região do Oeste Paulista por apresentar uma boa adaptação em condições climáticas, pedológicas e hídricas adversas, sendo que o ápice de desenvolvimento da mesma se dá em solos férteis ou corrigidos com adubação apropriada. Esta planta é utilizada principalmente em campos de pastagens. As braquiárias são espécies que têm elevado potencial de produção de forragem em solos férteis ou corrigidos com a adubação (SOARES FILHO, 1994).

Assim, o objetivo do presente estudo foi avaliar a capacidade de utilização do lodo da ETE em conjunto ou mesmo como alternativa à adubação química convencional na produção da braquiaria.

\section{METODOLOGIA}

O lodo de esgoto utilizado no experimento foi fornecido pela Companhia de Saneamento Básico do Estado de São Paulo SABESP, unidade de Presidente Prudente. O material adquirido é obtido por processo anaeróbico, sendo considerado lodo ativado, e classificado como classe $A$, segundo a Resolução CONAMA 375/2006.

Foram realizadas as análises de caracterização química do solo e do lodo para assim serem definidas as dosagens utilizadas nos tratamentos. Após o resultado das análises do lodo (Tabela 1), verificou-se que o mesmo apresenta boas condições de aplicabilidade no solo e ainda encontra-se dentro dos padrões aceitáveis da Resolução Federal CONAMA 375/2006 que dispõe sobre os critérios e procedimentos, para o uso agrícola de lodos de esgoto. 
Tabela 1. Caracterização do resíduo orgânico sólido obtido após a secagem.

\begin{tabular}{ccc}
\hline Parâmetros & Valores & Unidades \\
\hline $\mathrm{pH}$ & 7,6 & - \\
Umidade $65^{\circ} \mathrm{C}$ & 702,3 & $\mathrm{~g} \mathrm{~kg}^{-1}$ \\
MO Total & 83,4 & $\mathrm{~g} \mathrm{~kg}^{-1}$ \\
Carbono Orgânico & 46,3 & $\mathrm{~g} \mathrm{~kg}^{-1}$ \\
$\mathrm{C} / \mathrm{N}$ & 3,1 & - \\
$\mathrm{N}$ Total & 14,7 & $\mathrm{~g} \mathrm{~kg}^{-1}$ \\
Fósforo & 1,78 & $\mathrm{~g} \mathrm{~kg}^{-1}$ \\
Potássio & 1,3 & $\mathrm{~g} \mathrm{~kg}^{-1}$ \\
Cálcio & 19,5 & $\mathrm{~g} \mathrm{~kg}^{-1}$ \\
Magnésio & 1,0 & $\mathrm{~g} \mathrm{~kg}^{-1}$ \\
Enxofre & 0,6 & $\mathrm{~g} \mathrm{~kg}^{-1}$ \\
Cobre & 36,2 & $\mathrm{mg} \mathrm{kg}^{-1}$ \\
Ferro & $1.986,8$ & $\mathrm{mg} \mathrm{kg}^{-1}$ \\
Manganês & 68,8 & $\mathrm{mg} \mathrm{kg}^{-1}$ \\
Zinco & 74,9 & $\mathrm{mg} \mathrm{kg}^{-1}$ \\
\hline
\end{tabular}

Fonte: Autores, 2018.

Também foram coletadas dez sub amostras de solo para composição de uma única amostra para ser realizada análise química (Tabela 2) segundo a metodologia descrita por Raij et al. (2001).

Tabela 2. Caracterização dos atributos químicos do solo sem incorporação das dosagens de lodo.

\begin{tabular}{ccc}
\hline $\begin{array}{c}\text { Parâmetros } \\
\text { Químicos }\end{array}$ & Valores & Unidades \\
\hline $\mathrm{pH}\left(\mathrm{CaCl}_{2}\right)$ & 4,5 & - \\
$\mathrm{MO}$ & 6,3 & $\mathrm{~g} \mathrm{dm}^{-3}$ \\
$\mathrm{P}$ & 2,4 & $\mathrm{mg} \mathrm{dm}^{-3}$ \\
$\mathrm{~S}^{-\mathrm{SO}^{2-}}{ }_{4}$ & 7,5 & $\mathrm{mg} \mathrm{dm}^{-3}$ \\
$\mathrm{Al}^{3+}$ & 0,2 & $\mathrm{mmol}_{\mathrm{C}} \mathrm{dm}^{-3}$ \\
$\mathrm{H}+\mathrm{Al}$ & 20,6 & $\mathrm{mmol}_{\mathrm{C}} \mathrm{dm}^{-3}$ \\
$\mathrm{~K}$ & 1,7 & $\mathrm{mmol}_{\mathrm{C}} \mathrm{dm}^{-3}$ \\
$\mathrm{Ca}$ & 6,0 & $\mathrm{mmol}_{\mathrm{C}} \mathrm{dm}^{-3}$ \\
$\mathrm{Mg}$ & 1,3 & $\mathrm{mmol}_{\mathrm{C}} \mathrm{dm}^{-3}$ \\
$\mathrm{SB}$ & 9,0 & $\mathrm{mmol}_{\mathrm{C}} \mathrm{dm}^{-3}$ \\
$\mathrm{CTC}$ & 29,6 & $\mathrm{mmol}_{\mathrm{C}} \mathrm{dm}^{-3}$ \\
$\mathrm{M}$ & 2,2 & $\%$ \\
V & 30,3 & $\%^{\circ}$ \\
Boro & 0,36 & $\mathrm{mg} \mathrm{dm}^{-3}$ \\
Cobre & 0,60 & $\mathrm{mg} \mathrm{dm}^{-3}$ \\
Ferro & 0,90 & $\mathrm{mg} \mathrm{dm}^{-3}$ \\
Manganês & 0,70 & $\mathrm{mg} \mathrm{dm}^{-3}$ \\
Zinco & 0,20 & $\mathrm{mg} \mathrm{dm}^{-3}$ \\
\hline
\end{tabular}

Fonte: Autores, 2018.

Mediante a análise química verificou-se que o solo encontrava-se com $\mathrm{pH}$ ácido o que não é o ideal, pois as plantas têm dificuldades de sobreviver em solos com pH 4,0 e superior a 9,0, sendo que para a maioria delas desenvolver-se bem o pH do solo deve estar numa faixa entre 6 a 7,0 (KIEHL, 1979). O solo analisado ainda é classificado como de baixa 
fertilidade natural, pois o V\% encontra-se abaixo de $50 \%$.

Para cálculo da adubação a ser aplicada nos vasos, foi tomado como base à quantidade de nitrogênio (40 kg de $\mathrm{N} \mathrm{ha}^{-1}$ ) requerida pela braquiaria (RAlJ et al., 1997). Com base nesta necessidade e na concentração de $\mathrm{N}$ Kjeldahl do lodo foram definidas três doses, equivalentes a 0,$5 ; 1,0$ e 2,0 vezes a concentração de $\mathrm{N}$ requerido pela cultura. Para o desenvolvimento do trabalho estabeleceu-se 2 experimentos independentes, ambos com o delineamento experimental em blocos inteiramente casualizados, com cinco tratamentos e seis repetições (Tabela 3).

Tabela 3. Dosagens das adubações utilizadas em cada tratamento.

\begin{tabular}{ccc}
\hline $\begin{array}{c}\text { Tratamentos } \\
\text { (1) }\end{array}$ & $\begin{array}{c}\text { Dosagem } \\
\text { equivalente } \\
\text { de N }\end{array}$ & $\begin{array}{c}\text { Dosagem } \\
\text { equivalente } \\
\text { de N }\end{array}$ \\
\cline { 2 - 3 } & ${\text { (Kg ha- }{ }^{1} \text { ) }}^{\text {(mg/vaso) }}$ \\
\hline T1 & - & - \\
T2 & 40 & 628 \\
T3 & 20 e 20 & 314 e 968 \\
T4 & 40 & 1925 \\
T5 & 80 & 3850 \\
\hline
\end{tabular}

Fonte: Autores, 2018. ${ }^{(1)}$ T1=Testemunha; T2=100 \% N Mineral; T3=50 \% N Mineral e 50 \% N Orgânico; T4=100 \% N Orgânico; T5=200 \% N Orgânico.

No primeiro experimento os parâmetros de avaliação foram analisados com 100 dias após o plantio (DAP) e no segundo a avaliação dos parâmetros deu-se após 200 DAP.

Definidos os tratamentos, a condução do trabalho deu-se em vasos plásticos, preenchidos com $15 \mathrm{~kg}$ do solo coletado, sendo importante ressaltar que foi necessária a realização da calagem no solo (11 g), com a finalidade de corrigir a acidez do solo.

Após 30 dias da aplicação do calcário, realizou-se uma aplicação de super fosfato simples (fonte de $\mathrm{P}_{2} \mathrm{O}_{5}$ ), em todos os tratamentos na dosagem de $3.930 \mathrm{mg}$ por vaso, inclusive na testemunha. Posteriormente a este procedimento realizou-se a semeadura de 10 sementes de Urochloa decumbens por vaso. Após a germinação houve um raleio, permanecendo apenas 3 plantas por vaso.

Para proceder à avaliação do experimento na segunda e terceira etapa, após 100 e 200 DAP, foram coletados amostras da parte aérea da braquiaria para que assim fossem analisados os seguintes parâmetros: análise da produção de massa foliar e análise química da braquiaria. Após as coletas, os materiais foram enviados ao Laboratório de Análise de Solos e Tecido Vegetal e ao Laboratório de Análise Química e Física de Solos da Universidade do Oeste Paulista.

O primeiro procedimento realizado no laboratório foi à lavagem das folhas da braquiaria, de forma a evitar interferências de outros fatores no momento da pesagem, logo após as mesmas foram colocadas em cima da mesa para a secagem, sendo que esse processo demorou cerca de 20 horas, concluído o processo de secagem deu-se início a pesagem das folhas da braquiária, em balança eletrônica de precisão para obtenção do peso verde, ou seja, o peso antes da mesma ser enviada a estufa. Após a realização do peso verde, as folhas da planta foram enviadas para uma estufa de circulação forçada onde permaneceram por 72 horas numa temperatura de $65^{\circ} \mathrm{C}$, posteriormente a isto, o material seco foi submetido à pesagem em balança eletrônica de precisão para obtenção do peso seco (massa seca). 
Concluído procedimento anterior, deuse início ao processo de moagem das folhas da braquiaria, para ser submetido à análise foliar no Laboratório de Análise de Solos e Tecido Vegetal, seguindo a mesma metodologia de Malavolta et al. (1987).

Os dados obtidos foram submetidos à análise estatística, pelo programa ASSISTAT, e análises de regressão. Para comparação de médias, foi utilizado o teste Tukey (5\%).

\section{RESULTADOS E DISCUSSÕES}

Analisando a Tabela 4 verificou-se que o tratamento com maior peso verde e peso seco foi o T2, de forma geral pode-se dizer que foi o tratamento que obteve maior produção de massa verde e seca da planta, porém não houve significância estatística em relação às médias dos tratamentos.

Tabela 4. Análise descritiva das médias estatísticas de massa seca da parte aérea da braquiaria com 100 e 200 DAP.

\begin{tabular}{|c|c|c|c|c|c|c|}
\hline Amostra & $\begin{array}{c}\text { Med }^{*} \\
100 \\
\end{array}$ & DP & $\begin{array}{l}\text { CV } \\
(\%)\end{array}$ & $\begin{array}{r}\text { Med } \\
200 \\
\end{array}$ & DP & $\begin{array}{l}\text { CV } \\
(\%)\end{array}$ \\
\hline 1 & 4,63 & 8 & 4 & $\begin{array}{c}274,9 \\
8\end{array}$ & & 1 \\
\hline T2 & 9,23 & & & 14,68 & & 1 \\
\hline IT3 & 6,27 & 1,55 & 24 & $\begin{array}{c}352,8 \\
5\end{array}$ & & 96 , \\
\hline /T4 & 13 & 3,79 & 61,73 & $\begin{array}{c}323,7 \\
8\end{array}$ & & \\
\hline T5 & 37 & 4 & 3 & $\begin{array}{c}142,5 \\
2\end{array}$ & & 75 \\
\hline T1 & bu & J, & 32,76 & 31,67 & 5,16 & $1 \perp \perp$ \\
\hline & 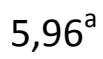 & & & & & 10,00 \\
\hline & $4,16^{a}$ & 1 & 24,19 & 35,82 & & 65,54 \\
\hline PST4 & $3,48^{a}$ & & 55,65 & 42,64 & 48,29 & 113,24 \\
\hline STS & $\underset{b}{2,54}$ & 1,62 & 63,67 & 20,4 & 12,08 & 59,25 \\
\hline
\end{tabular}

Fonte: Autores, 2018. PV= Peso Verde; PS= Peso Seco; DP= Desvio Padrão. *Médias, 100 e 200 dias, seguidas pela mesma letra, ou sem letra alguma, na coluna, não diferem significativamente entre si pelo teste Tukey a $5 \%$.

Ainda na Tabela 4 é possível verificar que na produção de massa verde o T3 atingiu maior valor, já na análise foliar de peso seco o T4 obteve o melhor resultado, valor com 200 DAP.

Fazendo um comparativo entre os resultados obtidos do peso verde e peso seco mostraram-se progressivos, porém em ambos o T5 manteve menor produtividade, tal situação contraria o estudo desenvolvido por Araujo, Gil e Tiritan (2009), onde eles constatam que o tratamento que recebeu maior dosagem de lodo $\left(80 \mathrm{mg}\right.$ de $\mathrm{N} \mathrm{dm}^{-3}$ de solo), proporcionou um aumento significativo na produção de massa seca da parte aérea.
De acordo com Kliemann (2003), essa variação na produção de massa da planta, ocorreu devido a fatores climáticos e temporais, pois em seu estudo constatou que o pico no desenvolvimento da braquiária deuse no terceiro ano após seu plantio. Outro autor que abordou tal assunto foi Tisdale, Nelson e Beaton (1985), encontrando altos índices de desenvolvimento foliar após 2 anos da aplicação de calcário no solo.

$\mathrm{Na}$ Tabela 5, observando-se os teores de Nitrogênio Total encontrado na parte aérea da planta, percebe-se que o T4 foi o que obteve a maior média dentre os demais, indicando desta forma que este foi o 
tratamento com o maior percentual de $\mathrm{N}$ disponível e absorvido pela braquiaria, entretanto não ocorreu significância estatística entre as amostras.

Tabela 5. Análise descritiva das médias estatísticas dos atributos químicos da massa seca da parte aérea da braquiaria com 100 e 200 DAP.

\begin{tabular}{|c|c|c|c|c|c|c|}
\hline Amostra & $\begin{array}{c}\text { Med }^{*} \\
100\end{array}$ & DP & $\begin{array}{l}\text { CV } \\
\text { (\%) }\end{array}$ & $\begin{array}{r}\text { Med }^{*} \\
200\end{array}$ & DP & $\begin{array}{l}\text { CV } \\
(\%)\end{array}$ \\
\hline N-Total $1\left(\mathrm{~g} \mathrm{Kg}^{-1}\right)$ & 14,25 & 2,50 & 17,50 & 10,17 & 4,03 & 39,70 \\
\hline N-Total $2\left(\mathrm{~g} \mathrm{Kg}^{-1}\right)$ & 14,95 & 2,70 & 17,49 & 9,01 & 4,16 & 46,09 \\
\hline N-Total $3\left(\mathrm{~g} \mathrm{Kg}^{-1}\right)$ & 13,76 & 2,57 & 18,70 & 11,45 & 0,94 & 8,24 \\
\hline N-Total $4\left(\mathrm{~g} \mathrm{Kg}^{-1}\right)$ & 22,7 & 4,55 & 20,06 & 10,77 & 2,86 & 26,55 \\
\hline N-Total $5\left(\mathrm{~g} \mathrm{Kg}^{-1}\right)$ & 11,6 & 1,80 & 12,55 & 12,38 & 3,88 & 31,37 \\
\hline P $1\left(\mathrm{~g} \mathrm{Kg}^{-1}\right)$ & 2,50 & 1,62 & 64,93 & 3,17 & 1,62 & 51,10 \\
\hline $\mathrm{P} 2\left(\mathrm{~g} \mathrm{Kg}^{-1}\right)$ & 1,73 & 0,64 & 37,61 & 3,53 & 0,38 & 10,70 \\
\hline P $3\left(\mathrm{~g} \mathrm{Kg}^{-1}\right)$ & 1,62 & 0,75 & 46,83 & 2,84 & 1,07 & 37,81 \\
\hline $\mathrm{P} 4\left(\mathrm{~g} \mathrm{Kg}^{-1}\right)$ & 1,22 & 0,07 & 5,74 & 2,65 & 1,28 & 48,66 \\
\hline P $5\left(\mathrm{~g} \mathrm{Kg}^{-1}\right)$ & 1,15 & 0,08 & 7,38 & 3,04 & 1,11 & 36,48 \\
\hline $\mathrm{K} 1\left(\mathrm{~g} \mathrm{Kg}^{-1}\right)$ & $20,9^{a}$ & 4,39 & 20,98 & 16,85 & 4,65 & 27,57 \\
\hline $\mathrm{K} 2\left(\mathrm{~g} \mathrm{Kg}^{-1}\right)$ & $\begin{array}{c}25,51 \\
a\end{array}$ & 3,60 & 14,13 & 11,63 & 3,27 & 28,10 \\
\hline $\mathrm{K} 3\left(\mathrm{~g} \mathrm{Kg}^{-1}\right)$ & $\underset{a}{25,12}$ & 5,81 & 23,16 & 12,27 & 1,73 & 14,12 \\
\hline $\mathrm{K} 4\left(\mathrm{~g} \mathrm{Kg}^{-1}\right)$ & $4,12^{b}$ & 2,57 & 62,45 & 15,12 & 5,16 & 34,16 \\
\hline $\mathrm{K} 5\left(\mathrm{~g} \mathrm{Kg}^{-1}\right)$ & $\underset{b}{13,45}$ & 3,98 & 29,61 & 16,41 & 5,16 & 34,17 \\
\hline Ca $1\left(\mathrm{~g} \mathrm{Kg}^{-1}\right)$ & $3,5^{b}$ & 0,58 & 16,65 & $1,5^{b}$ & 0,37 & 25,30 \\
\hline Ca $2\left(\mathrm{~g} \mathrm{Kg}^{-1}\right)$ & $1,38^{b}$ & 0,82 & 59,18 & $1,67^{b}$ & 1,03 & 61,96 \\
\hline Са $3\left(\mathrm{~g} \mathrm{Kg}^{-1}\right)$ & $1,18^{b}$ & 0,56 & 47,32 & $2,07^{b}$ & 0,61 & 29,62 \\
\hline Ca $4\left(\mathrm{~g} \mathrm{Kg}^{-1}\right)$ & $0,4^{c}$ & 0,1 & 25,00 & $2,97^{\mathrm{a}}$ & 0,94 & 31,95 \\
\hline Ca $5\left(\mathrm{~g} \mathrm{Kg}^{-1}\right)$ & $\begin{array}{c}13,45 \\
a\end{array}$ & 3,98 & 29,62 & $3,78^{\mathrm{a}}$ & 0,97 & 25,81 \\
\hline $\operatorname{Mg} 1\left(\mathrm{~g} \mathrm{Kg}^{-1}\right)$ & $4,55^{b}$ & 0,58 & 12,75 & 3,18 & 0,39 & 12,15 \\
\hline $\operatorname{Mg} 2\left(\mathrm{~g} \mathrm{Kg}^{-1}\right)$ & $4,24^{b}$ & 0,58 & 13,81 & 3,12 & 0,35 & 11,37 \\
\hline $\operatorname{Mg} 3\left(\mathrm{~g} \mathrm{Kg}^{-1}\right)$ & $4,76^{b}$ & 0,79 & 16,78 & 3,67 & 0,61 & 16,60 \\
\hline $\operatorname{Mg} 4\left(\mathrm{~g} \mathrm{Kg}^{-1}\right)$ & $5,57^{a}$ & 0,30 & 5,49 & 3,47 & 0,60 & 17,32 \\
\hline $\operatorname{Mg} 5\left(\mathrm{~g} \mathrm{Kg}^{-1}\right)$ & $4,55^{b}$ & 0,35 & 7,77 & 3,81 & 0,67 & 17,60 \\
\hline $\mathrm{S} 1\left(\mathrm{~g} \mathrm{Kg}^{-1}\right)$ & $1,12^{\mathrm{a}}$ & 0,12 & 11,18 & 1,3 & 0,36 & 27,95 \\
\hline $\mathrm{S} 2\left(\mathrm{~g} \mathrm{Kg}^{-1}\right)$ & $0,96^{b}$ & 0,7 & 25,08 & 1,05 & 0,32 & 30,56 \\
\hline $\mathrm{S} 3\left(\mathrm{~g} \mathrm{Kg}^{-1}\right)$ & $0,98^{b}$ & 0,13 & 13,30 & 1,17 & 0,20 & 16,85 \\
\hline $\mathrm{S} 4\left(\mathrm{~g} \mathrm{Kg}^{-1}\right)$ & $0,96^{b}$ & 0,29 & 29,86 & 1,12 & 0,245 & 22,24 \\
\hline $\mathrm{S} 5\left(\mathrm{~g} \mathrm{Kg}^{-1}\right)$ & $0,8^{b}$ & 0 & 0 & 1,25 & 0,27 & 21,91 \\
\hline $\mathrm{Cu} 1\left(\mathrm{mg} \mathrm{Kg}^{-1}\right)$ & $10,4^{a}$ & 5,17 & 49,69 & 11,03 & 5,18 & 46,92 \\
\hline $\mathrm{Cu} 2\left(\mathrm{mg} \mathrm{Kg}^{-1}\right)$ & $7,94^{\mathrm{a}}$ & 3,77 & 47,53 & 10,6 & 2,93 & 27,66 \\
\hline $\mathrm{Cu} 3\left(\mathrm{mg} \mathrm{Kg}^{-1}\right)$ & $8,5^{a}$ & 4,97 & 58,43 & 18,2 & 6,52 & 35,83 \\
\hline $\mathrm{Cu} 4\left(\mathrm{mg} \mathrm{Kg}^{-1}\right)$ & $7,13^{a}$ & 0,81 & 11,42 & 14,93 & 7,25 & 48,55 \\
\hline $\mathrm{Cu} 5\left(\mathrm{mg} \mathrm{Kg}^{-1}\right)$ & $4,25^{b}$ & 1,77 & 41,59 & 17,57 & 4,37 & 24,86 \\
\hline
\end{tabular}

Fonte: Autores, 2018. DP= Desvio Padrão. 'Médias, 100 e 200 dias, seguidas pela mesma letra, ou sem letra alguma, na coluna, não diferem significativamente entre si pelo teste Tukey a $5 \%$. 
Tabela 5. Análise descritiva das médias estatísticas dos atributos químicos da massa seca da parte aérea da braquiária com 100 e 200 DAP. (Continuação)

\begin{tabular}{|c|c|c|c|c|c|c|}
\hline Amostra & $\begin{array}{c}\text { Med }^{*} \\
100\end{array}$ & DP & $\begin{array}{l}\text { CV } \\
(\%)\end{array}$ & $\begin{array}{c}\text { Med }^{*} \\
200\end{array}$ & DP & $\begin{array}{l}\text { CV } \\
(\%)\end{array}$ \\
\hline Fe $1\left(\mathrm{mg} \mathrm{Kg}^{-1}\right)$ & $419,67^{a}$ & 6,43 & 5,37 & 163,98 & 159,73 & 97,40 \\
\hline Fe $2\left(\mathrm{mg} \mathrm{Kg}^{-1}\right)$ & $84,04^{b}$ & 42,45 & 50,51 & 68,48 & 37,17 & 54,27 \\
\hline Fe $3\left(\mathrm{mg} \mathrm{Kg}^{-1}\right)$ & $38,86^{b}$ & 13,73 & 35,33 & 92,17 & 68,94 & 74,80 \\
\hline Fe $4\left(\mathrm{mg} \mathrm{Kg}^{-1}\right)$ & $43,83^{b}$ & 40,24 & 91,81 & 90,67 & 63,08 & 69,57 \\
\hline Fe $5\left(\mathrm{mg} \mathrm{Kg}^{-1}\right)$ & $81,7^{b}$ & 63,64 & 77,89 & 128,65 & 96,25 & 74,81 \\
\hline $\operatorname{Mn} 1\left(\mathrm{mg} \mathrm{Kg}^{-1}\right)$ & $120,35^{c}$ & 31,77 & 26,40 & 81,37 & 57,18 & 70,28 \\
\hline $\operatorname{Mn} 2\left(\mathrm{mg} \mathrm{Kg}^{-1}\right)$ & $117,7^{\mathrm{bc}}$ & 28,71 & 23,73 & 58,47 & 27,89 & 47,70 \\
\hline $\operatorname{Mn~} 3\left(\mathrm{mg} \mathrm{Kg}^{-1}\right)$ & $153,7^{b}$ & 33,21 & 21,61 & 56,33 & 13,97 & 24,81 \\
\hline $\operatorname{Mn} 4\left(\mathrm{mg} \mathrm{Kg}^{-1}\right)$ & $176,9^{a}$ & 19,93 & 11,27 & 55,87 & 25,50 & 45,65 \\
\hline $\operatorname{Mn} 5\left(\mathrm{mg} \mathrm{Kg}^{-1}\right)$ & $182,8^{a}$ & 20,36 & 11,14 & 60,82 & 41,19 & 67,73 \\
\hline $\mathrm{Zn} 1\left(\mathrm{mg} \mathrm{Kg}^{-1}\right)$ & $196,45^{a}$ & 125,42 & 63,84 & 29,78 & 15,14 & 50,83 \\
\hline $\mathrm{Zn} 2\left(\mathrm{mg} \mathrm{Kg}^{-1}\right)$ & $\left\lfloor 30,22^{b}\right.$ & 79,68 & 61,84 & 19,53 & 15,16 & 77,60 \\
\hline $\mathrm{Zn} 3\left(\mathrm{mg} \mathrm{Kg}^{-1}\right)$ & $68,22^{c}$ & 69,80 & 102,33 & 23,25 & 5,20 & 22,35 \\
\hline $\mathrm{Zn} 4\left(\mathrm{mg} \mathrm{Kg}^{-1}\right)$ & $89,83^{c}$ & 73,86 & 82,22 & 29,73 & 19,85 & 66,77 \\
\hline $\mathrm{Zn} 5\left(\mathrm{mg} \mathrm{Kg}^{-1}\right)$ & $182,8^{a}$ & 20,36 & 11,14 & 33,52 & 13,08 & 39,03 \\
\hline
\end{tabular}

Fonte: Autores, 2018. DP= Desvio Padrão. "Médias, 100 e 200 dias, seguidas pela mesma letra, ou sem letra alguma, na coluna, não diferem significativamente entre si pelo teste Tukey a $5 \%$.

Já para o potássio $(\mathrm{K})$ encontrado na parte aérea da braquiária, os melhores resultados em comparação aos demais, foram no T1, T2 e T3, demonstrando significância estatística em relação aos demais. Nas amostras com apenas adição de lodo no solo, pode-se perceber que ocorreu a redução nos teores de potássio, situação encontrada por Araujo, Gil e Tiritan (2009).

Para o cálcio ( $\mathrm{Ca}$ ) encontrado na parte aérea da braquiária, o T5 obteve relevância estatística em relação aos outros tratamentos, sendo que o pior resultado encontrado foi no T4.

No caso do magnésio $(\mathrm{Mg})$ o T4 foi o de maior relevância estatística, revelando que este tratamento teve maior índice de magnésio absorvido pelas folhas da braquiária.

Com o enxofre (S) o tratamento que mais destacou- se estatisticamente foi o T1, tendo um pior resultado de absorção nos demais tratamentos.

Outra análise realizada foi a do cobre (Cu), onde percebe- se que o $\mathrm{T} 1, \mathrm{~T} 2, \mathrm{~T} 3, \mathrm{~T} 4$ se destacaram com maior absorção do elemento nas folhas da braquiária, onde apenas o T5 que continha adubação de $200 \%$ N Orgânico teve um menor resultado comparado aos demais.

Para o ferro (Fe) o T1 foi o que se sobressaiu estatisticamente, tratamento que representa solo in natura, sem nenhum tipo de adubação, os demais tratamentos não tiveram relevância entre eles.

Já para o zinco $(Z n)$ o T1 e o T5 destacaram-se estatisticamente e os piores resultados foram obtidos nos T3 e T4 tendo uma máxima e um mínimo inferior aos demais tratamentos.

Ainda analisando a Tabela 5, observouse que o único elemento que obteve relevância estatística com as análises feitas após os 200 DAP foi o cálcio, demonstrando maior resultado no $\mathrm{T} 4$ e $\mathrm{T5}$, tratamentos que continham apenas adubo orgânico, os demais não tiveram relevância estatística entre eles.

Já o ferro apresentou as maiores médias no T1 e T5, demonstrando maior absorção deste elemento na parte aérea da braquiária.

No entanto, as demais análises de $\mathrm{N}$ Total, $\mathrm{P}, \mathrm{K}, \mathrm{Mg}, \mathrm{S}, \mathrm{Cu}, \mathrm{Mn}$ e $\mathrm{Zn}$ não apresentaram significância estatística. 


\section{CONSIDERAÇÕES FINAIS}

Mediante os resultados já discutidos, verificou-se que não houve diferença entre a adubação química convencional e a adubação orgânica, o mesmo ocorreu entre 0 tratamento testemunha no presente trabalho de análise dos atributos da parte aérea da braquiária, isso sucedeu possivelmente devido a interferência da calagem, pois a mesma ocultou a real eficiência dos tratamentos.

Com isso conclui-se que a adubação orgânica se equivaleu com a adubação química.

\section{REFERÊNCIAS}

ALAMINO, R. C. J. A utilização do lodo de esgoto como alternativa sustentável na recuperação de solos degradados: viailidade, avaliação e biodisponibilidade de metais. 2010. Tese (Doutorado em Geologia) Instituto de Geociências da Universidade Federal do Rio de Janeiro (UFRJ), Rio de Janeiro, 2010.

ANDREOLI, C. V.; PEGORINI, E. S.; FERNANDES, F. Disposição do lodo de esgoto no solo. In: ANDREOLI, C. V.; Von SPERLING, M.; FERNANDES, F. (coords). Lodo de esgotos: Tratamento e disposição final. Belo Horizonte: Universidade Federal de Minas Gerais DESA/UFMG, SANEPAR, 2001..

ARAUJO, F. F.; GIL, F. C; TIRITAN, C. S. Lodo de esgoto na fertilidade do solo, na nutrição de Brachiaria decumbens e na atividade da desidrogenase. Pesquisa Agropecuária Tropical, v.39, n. 1, 2009.

BRASIL. RESOLUÇÃO CONAMA no 375, de 29 de agosto de 2006. Define critérios e procedimentos, para o uso agrícola de lodos de esgoto gerados em estações de tratamento de esgoto sanitário e seus produtos derivados, e dá outras providências. Diário Oficial da União, Brasília, DF, 29 de Ago. de 2006. Disponível em: http://www.mma.gov.br/port/conama/res/res 06/res37506.pdf. Acesso: 15 jun. 2018.
KIEHL, J. C. Manual de edafologia: relação solo planta. São Paulo: Agronômica Ceres, 1979.

KLIEMANN, H. J. Relações da produção de massa verde de brachiaria brizantha com os índices de disponibilidade de nutrientes em solos sob o sistema barreirão de manejo. Agricultural Research in the Tropics, v. 33, n. 1, p. 49-56, 2003.

MALAVOLTA, E. et al. Acumulação de matéria seca e de micronutrientes nos frutos de cinco variedades de citros durante $O$ seu crescimento. In: CONGRESSO BRASILEIRO DE FRUTICULTURA, 9., 1987, Campinas. Anais [...]. Campinas: Sociedade Brasileira de Fruticultura, 1987. v.1

NASCIMENTO, P. C. D; LANI J. L; MENDONÇA, E. S; ZOFFOLI, H. J. O; PEIXOTO, H. T. M. Teores e características da matéria orgânica de solos hidromórficos do Espírito Santo. Rev. Bras. Ciênc. Solo [online],v.34, n.2, p.339-348, 2010.

RAIJ, B. Van; ANDRADE, J. C; CANTARELLA, H; QUAGGIO, J. A. Análise química para avaliação da fertilidade de solos tropicais. Campinas: Instituto Agronômico de Campinas, 2001.

RAIJ, B. Van; CANTARELLA, H; QUAGGIO, J. A; FURLANI, A. M. C. (Eds.) Recomendações de adubação e calagem para o Estado de São Paulo. 2.ed.rev.atual. Campinas: IAC, 1997.

SOARES FILHO, C. V. Recomendação de espécies e variedades de Brachiaria para diferentes condições. In: SIMPÓSIO SOBRE MANEJO DA PASTAGEM, Piracicaba, 1994 Anais [...]. Piracicaba: USP, 1994.

TISDALE, S. L.; NELSON, W. L.; BEATON, J. D. Soil fertility and fertilizers. 4. ed. New York: Mc Millan, 1985. 754 p.

TSUTIYA, M. T. Alternativas de disposição final de biossólidos gerados em estações de tratamento de esgotos. In: BETTIOL, W.; CAMARGO, O. A. Impacto ambiental do uso 
agrícola do lodo de esgoto. Jaguariúna: EMBRAPA, 2000.

VON SPERLING, M.; GONÇALVES, R. F. Lodo de esgotos: características e produção. In: ANDREOLI, C.V.; VON SPERLING, M.; FERNANDES, F. (Org.) Lodo de esgotos: tratamento e disposição final. Belo Horizonte: Departamento de Engenharia Sanitária e Ambiental, UFMG; Curitiba: SANEPAR, 2001. 484 p. (Princípios do tratamento biológico de águas residuárias, v. 6. cap. 2, p. 17-67). 